\title{
Redução De Consumo De Energia Em Sistemas De Telefonia Comunitária Usando Raspberry Pi
}

\author{
Gabriel Couto \\ gabriel.couto@itec.ufpa.br \\ Universidade Federal do Pará
}

\author{
Giovanni Leão \\ giovanni.laredo@itec.ufpa.br \\ Universidade Federal do Pará
}

\author{
Lauro Castro \\ lauro.castro@ufpa.br \\ Universidade Federal do Pará
}

\author{
Francisco Muller \\ fmuller@ufpa.br \\ Universidade Federal do Pará
}

\author{
Aldebaro Klautau \\ aldebaro@ufpa.br \\ Universidade Federal do Pará
}

\begin{abstract}
This article presents an energy consumption analysis of two different emulation scenarios of a GSM (Global System for Mobile) network; one with a traditional computer and another with Raspberry Pi 3. The network is created by open-source software that emulates the main modules of a GSM network, this emulation combined with a radio generator equipment enables calls and message exchange between network users. The CELCOM project designs and implements community networks in digitally isolated regions, the scenarios presented in this article can be applied in works of CELCOM project. The power distribution service of this GSM network, in isolated regions, does not have the required stability to maintain the quality in community networks, creating a demand for an alternative power system. Different scenarios are proposed and analyzed in this work, trying to get a lower energy consumption.
\end{abstract}

\section{KEYWORDS}

Telecommunications, GSM, Community networks, Raspberry pi, Low cost

\section{INTRODUÇÃO}

Com a tendência da globalização do mundo atual, a inclusão digital se torna umas das principais ferramentas de inserção do indivíduo na sociedade moderna. No Brasil, o acesso de domicílios à internet e à telefonia vem crescendo e em 2018 aproximadamente $63 \%$ dos domicílios da região amazônica possuem acesso a estes serviços de telecomunicações [1]. Contudo, ainda há comunidades nas quais o acesso à tecnologia é raso e há grandes dificuldades para prover acesso à informação e comunicação nessas localidades. Por esses motivos os projetos de telefonia comunitária se fazem necessários para desenvolver e facilitar a comunicação entre essas comunidades e o mundo globalizado.

Em muitas regiões isoladas o fornecimento de energia elétrica, quando não ausente, é precário e esta baixa qualidade prejudica o funcionamento dos principais equipamentos de telecomunicações. Este torna-se um dos fatores que contribuem com atraso do desenvolvimento dessas localidades. Visto a necessidade de sistemas alternativos de fornecimento de energia elétrica que asseguram o bom funcionamento de equipamentos com alta eficiência e com baixo consumo energético, este artigo busca propor e analisar dois cenários de uma rede de segunda geração de telefonia móvel (2G) comunitária com baixo consumo de energia. As próximas seções estão organizadas da seguinte forma: na Seção 2 é explicado o projeto de telefonia comunitária do CELCOM e são expostos os softwares utilizados para criar a rede GSM. A seção 3 descreve os cenários propostos, enquanto na seção 4 o método de análise de consumo utilizado é descrito. Por fim, na Seção 5 temos as conclusões finais do artigo.

\section{PROJETO CELCOM E SOFTWARES QUE EMULAM A REDE GSM}

O projeto CELCOM tem como foco desenvolver e implantar tecnologias de baixo custo em redes comunitárias. Sendo um projeto de cunho tecnológico e social, o CELCOM busca implementar os projetos desenvolvidos em laboratório nas comunidades isoladas do estado do Pará. Atualmente o CELCOM atende duas comunidades onde provê acesso à internet e telefonia. Em ambas regiões alvo do projeto, a rede de telefonia é afetada por oscilações da rede de energia elétrica. Situações como queima de equipamentos e o mal funcionamento do sistema de telefonia devido à quedas de tensão se tornaram constantes, o que pode até inviabilizar o projeto e o processo de inclusão digital destas localidades.

Em ambos cenários propostos foram instalados, em uma distribuição baseada no Debian 8, softwares open-source da Osmocom [2]. Estes formam a parte da BSC (Base Station Controller) que é responsável pela gerência da rede GSM. Os módulos instalados ainda criam suporte para a BTS (Base Transceiver Station), gerando assim um ambiente completo.

A rede criada possibilita a comunicação por voz e a troca de mensagens de texto entre os usuários da mesma. Ainda é possível adicionar um provedor VOIP (Voice Over IP), que possibilita a ligação entre os usuários locais e usuários de outras redes de telefonia.

\section{CENÁRIOS PROPOSTOS}

No cenário A, a BSC é emulada por um computador munido de um processador intel Core i5, 8 Gigabytes de memória $R A M$ e alimentado por uma fonte EVGA 500B com certificado 80 plus [3]. No cenário B, a BSC é composta por um Raspberry Pi 3 model B [4] com o processador Broadcom BCM 2837 de 1.2 Gigahertz com 4 núcleos ARM Cortex-A53, 1 Gigabyte de memória RAM sendo alimentado por uma fonte CanaKit DCAR-052A5 [5]. O hardware USRP (Universal Software Radio Peripheral), modelo B100 [6], está presente nos dois cenários. Este, tem a função de BTS e possui uma fonte de alimentação de tensão contínua de 6 Volts e 3 Amperes na saída. A Figura 1 mostra os dois cenários propostos. 


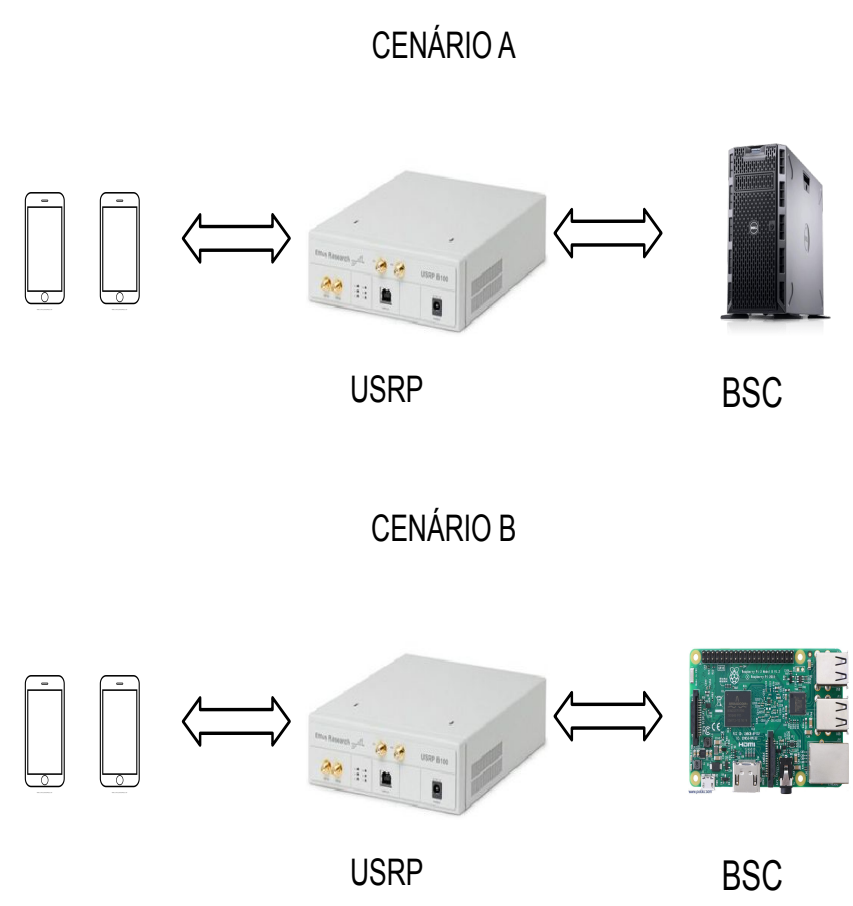

Figure 1: Cenários

\section{COLETA E TRATAMENTO DE DADOS}

Para realizar a análise do consumo dos equipamentos nos dois cenários propostos, a tensão e corrente eficazes foram coletadas utilizando um voltímetro e um amperímetro. Com os valores de tensão e corrente obtidos, multiplicamos esses valores e encontramos a potência aparente (S) do circuito em cada instante. Para os cálculos futuros, iremos utilizar a média aritmética desses valores. Estes passos são descritos pela Equação 1, onde $S$ é a média da potência aparente, $\mathrm{N}$ é o número de amostras e $\mathrm{V}_{\mathrm{ef}}$ e $\mathrm{I}_{\mathrm{ef}}$ são a tensão e correntes eficazes, respectivamente. É importante ressaltar que apenas o consumo da BSC de cada cenário foi analisado.

$$
S=\frac{1}{N} \times \sum_{k=1}^{N} V_{e f_{k}} \times I_{e f_{k}}
$$

Para obter a potência ativa $(\mathrm{P})$ a partir da potência aparente, se faz necessário o uso do fator de potência (FP) do equipamento que está sendo analisado e da Equação 2 [7]. O fator de potência das fontes é de 0.98 , devido ao PFC (power factor correction) ativo e as características intrínsecas das fontes nos dois casos.

$$
P=S \times F P
$$

A Tabela 1 mostra os valores obtidos após a coleta e tratamento dos dados. O resultado é dado em Watts, unidade da potência ativa, e para obter o consumo do sistema em um período de tempo $T$, utiliza-se a Equação 3.

$$
E=\frac{P}{1000} \times T
$$

O consumo E possui a unidade de $k W H$, essa sendo a unidade mais utilizada nas medidas de consumo pelas distribuidoras de energia. Como o sistema tem como proposta realizar um serviço fundamental para a comunidade, deve-se fazer os cálculos para um funcionamento integral, 24 horas por dia e 30 dias no mês, como é mostrado na terceira coluna da Tabela 1.

Table 1: Dados Obtidos

\begin{tabular}{ccc}
\hline Dispositivo & Potência Ativa(W) & Consumo Mensal(kWH) \\
\hline Computador & 24.696 & 17.781 \\
Raspberry Pi 3 & 4.2 & 3.024 \\
\hline
\end{tabular}

\section{CONCLUSÃO}

A partir da análise da potência que cada cenário necessita, concluímos que o cenário B é o mais adequado para projetos de telefonia comunitária em regiões isoladas visto que por demandar pouca energia, sistemas alternativos de alimentação elétrica são mais baratos. Além disso, por sua pequena dimensões o cenário B é uma aplicação flexível e escalonável.

O cenário B se difere nos custos relacionados aos equipamentos, operação e manutenção criando uma solução atrativa para redes de telefonia comunitária. Além de promover a inclusão social e digital, o projeto CELCOM possibilita o estudo e o desenvolvimento de várias novas tecnologias adaptadas à realidade das comunidades esparsamente povoadas através de uma rede comunitária.

\section{AGRADECIMENTOS}

Os Autores agradecem a todos os participantes do CELCOM. O financiamento para os pilotos do CELCOM é da Secretaria de Ciência, Tecnologia e Educação Técnica do Estado do Pará (PRODEPA), CAPES e Fundação de Amparo à pesquisa do Estado de São Paulo (FAPESP), número do processo 2015/24508-9

\section{REFERÊNCIAS}

[1] Cetic. Tic domicílios: Proporção de domicílios com acesso à internet, October 2018. URL https://cetic.br/media/analises/tic_domicilios_2018_coletiva_de_ imprensa.pdf.

[2] Osmocom. Cellular network infrastructure, October 2019. URL https://osmocom. org/projects/cellular-infrastructure.

[3] Evga. Evga 500b, November 2013. URL https://br.evga.com/articles/00763/.

[4] Raspberry Pi Foundation. Raspberry pi 3 model b, October 2019. URL https://www.raspberrypi.org/products/raspberry-pi-3-model-b/.

[5] Canakit. Raspberry pi 3 power supply - 2.5a (micro usb), October 2019. URL https://www.canakit.com/raspberry-pi-adapter-power-supply-2-5a.html.

[6] Ettus Research. B100, October 2016. URL https://kb.ettus.com/B100.

[7] Matthew Sadiku Charles K. Alexander. Fundamentos de Circuitos Elétricos. BookMan, 5st edition, 2013. 